\title{
Comparison of Physicochemical Properties of Nano- and Microsized Crystals in the Urine of Calcium Oxalate Stone Patients and Control Subjects
}

\author{
Jie Gao, ${ }^{1}$ Jun-Fa Xue, ${ }^{2}$ Meng Xu, ${ }^{2}$ Bao-Song Gui, ${ }^{1}$ Feng-Xin Wang, ${ }^{2}$ and Jian-Ming Ouyang ${ }^{2}$ \\ ${ }^{1}$ Department of Nephrology, The Second Hospital of Xian Jiaotong University, Xian 710004, China \\ ${ }^{2}$ Institute of Biomineralization and Lithiasis Research, Jinan University, Guangzhou 510632, China \\ Correspondence should be addressed to Bao-Song Gui; guibsdoctor@sina.com and Jian-Ming Ouyang; toyjm@jnu.edu.cn
}

Received 20 March 2014; Revised 5 April 2014; Accepted 8 April 2014; Published 30 April 2014

Academic Editor: Renyun Zhang

Copyright (C) 2014 Jie Gao et al. This is an open access article distributed under the Creative Commons Attribution License, which permits unrestricted use, distribution, and reproduction in any medium, provided the original work is properly cited.

\begin{abstract}
Purpose. To compare the properties of different sizes of urinary crystallites between calcium oxalate $(\mathrm{CaO} x)$ calculi patients and healthy controls. Methods. We studied the average particle size, size distribution, intensity-autocorrelation curve, zeta potential $(\zeta)$, conductivity, mobility, aggregation state, and stability of different sizes of urinary crystallites by nanoparticle size analysis and transmission electron microscopy after filtration through a microporous membrane with an aperture size from $0.22 \mu \mathrm{m}$ to $0.45,1.2,3$, and $10 \mu \mathrm{m}$. Results. The urinary crystallites of the $\mathrm{CaO} x$ calculi patients were uneven and much easy to aggregate than those of controls. The number of large-sized crystallites of the patients was significantly more than that of the controls. The main components of the nanosized urinary crystallites in patients were $\mathrm{CaO} x$ monohydrate (COM), uric acid, and $\beta$-calcium phosphate, and these components were basically similar to those of the microsized urinary crystallites. The urinary crystallites of the calculi patients were easier to aggregate than that of the controls, and the small-sized urinary crystallites were much easier to agglomerate. Conclusions. The urinary system of $\mathrm{CaO} x$ calculi patients is unstable and highly susceptible to urinary crystallite aggregation. The rapid aggregation of urinary crystallites may be the key factor affecting urolithiasis formation.
\end{abstract}

\section{Introduction}

The formation of kidney stones is closely related to the crystallites in the urine [1-3]. The agglomeration and growth of crystallites in urine are two main factors affecting the growth of urinary stone [4]. Urine supersaturation leads to nucleation of insoluble salt (nanosized, generally $<10 \mathrm{~nm}$ ) and subsequent growth or aggregation of the crystal nucleus into crystallites of pathological size (microns or dozens of microns). After adhesion to the renal epithelial cells [5], urinary crystallites would gradually grow and finally form urinary stones (millimeter level, generally ranging from a few millimeters to a few centimeters) [6]. Therefore, several studies [7-9] believed that urolithiasis was more accurate to be predicted by crystalluria than $24 \mathrm{~h}$ urine volume or calcium excretion and even more accurate than urinary calcium and urinary oxalate concentration.
Different sizes of urinary crystallites have been observed, such as crystallites with a size of $1 \mathrm{~nm}$ to $1000 \mathrm{~nm}[6,10-13]$ and that more than tens of microns $[14,15]$. However, whether the effect of these different sizes of urinary crystallites on urinary stone formation differs or not remains unknown. Thus, the evaluations of the physicochemical properties of micro- and nanocrystallites in urines of calcium oxalate $(\mathrm{CaO} x)$ calculi patients and healthy controls may have potential clinical value because if the properties of urinary crystallites were reliable predictor of disease, such a method would represent a noninvasive method that could potentially by applied.

Different pore sizes $(0.22,0.45,1.2,3$, and $10 \mu \mathrm{m})$ of microporous membranes were used to filter the urine of $10 \mathrm{CaO} x$ stone formers and 10 healthy controls to obtain differently sized (from nanometers to micrometers) urinary crystallites. The properties of the different size ranges of 
urinary crystallites, including average particle size $(\bar{d})$, size distribution, intensity-autocorrelation curve, zeta potential $(\zeta)$, conductivity, mobility, crystallites components, morphology, and aggregation state, were compared to clarify the differences of nucleation, growth, and aggregation of different sizes of urinary crystallites between urolithiasis patients and healthy controls. The results could provide relevant information to elucidate the mechanism for the formation of urolithiasis.

\section{Experiment}

2.1. Reagents and Instruments. Absolute ethanol, sodium azide $\left(\mathrm{NaN}_{3}\right)$, and all the reagents were analytical purity. All glass vessels were cleaned with double distilled water. Microporous membrane (pore size: $0.22,0.45,1.2,3$, and $10 \mu \mathrm{m}$, resp.) was purchased from Xinya company (Shanghai, China).

The size distribution and zeta potential of crystallites in urine were determined by nanoparticle size analyzer of Zetasizer Nano-ZS (Malvern, England). The experimental conditions were as follows: incident beam of the $\mathrm{He}-\mathrm{Ne}$ laser, $\lambda=633.0 \mathrm{~nm}$, and the incident angle of $90^{\circ}$. The samples were observed with a TECNAI-10 transmission electron microscope (Philips, The Netherlands) running at accelerating voltage of $100 \mathrm{kV}$. Image Pro Plus 5.02 (Media Cybernetics, USA) was used to analyze the diameter and count the number of particles in the TEM images. X-ray diffraction (XRD) results were recorded on a D/max- $\gamma \mathrm{A} X-$ ray diffractometer (Rigaku, Japan), using Ni-filtered $\mathrm{Cu}-\mathrm{K} \alpha$ radiation $(\lambda=1.54 \AA)$ and a scanning rate of $2^{\circ} \mathrm{min}^{-1}$ at $40 \mathrm{kV}, 30 \mathrm{~mA}$, in the $2 \theta$ range of $5^{\circ}$ to $60^{\circ}$, the divergence and scattering slit was $1^{\circ}$. Centrifugalization was carried by 80-1 sedimentation centrifuge (Shanghai Surgical Instrument Factory, China).

2.2. Collection and Treatment of Urine. The participants in the study included 10 calculi patients with $\mathrm{CaO} x$ stones (seven men and three women; mean age $=50.2$ years; range $=$ 28 68 years) and 10 healthy persons without prior history of urinary stones ( 37.2 years; range $=23 \sim 57$ years). The calculi patients were from the Lithotripsy Center of the First Affiliated Hospital of Jinan University, 10 healthy subjects were from the students and teachers of Jinan University, all of them are Chinese. The study was approved by the Institutional Review Board of the First Affiliated Hospital of Jinan University, and all participants provided informed consent.

Fasting morning urines were collected. After the $\mathrm{pH}$ value was detected, $1 \% \mathrm{NaN}_{3}$ solution $(10 \mathrm{~mL} / \mathrm{L}$ urine sample) was added into these urine samples as antiseptic. Subsequently, $20 \mathrm{~mL}$ anhydrous alcohol was added to $30 \mathrm{~mL}$ of urine sample. The solution was stirred for $3 \mathrm{~min}$ and left undisturbed for half an hour. Then, the above treated urine was filtered by microporous membrane with different pore sizes $(0.22,0.45$, $1.2,3$, and $10 \mu \mathrm{m})$ to remove the proteins and cell debris in the urines. The filtered urine was stored in clean glassware for detecting. After such a series of treatment, the influence of food metabolism was eliminated.
2.3. Measurement of Urinary Crystallites. After ultrasonication of the above filtered urines for $3 \mathrm{~min}$, the nanoparticle size analyzer was immediately used to detect the average particle size, particle size distribution, migration, conductivity, autocorrelation curve, polydispersity index (PDI), and $\zeta$ of the urinary nanocrystallites. All the data were the average values of three parallel tests. The experimental data were expressed as the mean \pm standard deviation $(\bar{x} \pm s)$.

2.4. Transmission Electron Microscopy (TEM) Detection of Urinary Crystallites. After ultrasound treatment of the urine sample for $3 \mathrm{~min}, 3 \mu \mathrm{L}$ of urine was dipped into a copper mesh by a microsyringe and stored in a desiccator for 1 day prior to examination by TEM.

\section{Results and Discussion}

3.1. Size Distribution and Average Particle Diameter Differences. The size distribution and average particle diameter of two types of urinary crystallites from 10 cases of $\mathrm{CaO} x$ calculi patients and 10 cases of healthy subjects after filtration through microporous membranes with different pore sizes were comparatively studied as follows.

(1) The $\bar{d}$ values of the urinary crystallites of 10 calculi patients increased by approximately $2700 \mathrm{~nm}$ from about $282 \mathrm{~nm}$ to $2953 \mathrm{~nm}$ when the pore size of the microporous membranes increased from $0.22 \mu \mathrm{m}$ to $10 \mu \mathrm{m}$ (Figure 1(a)). By contrast, the $\bar{d}$ values of the urinary crystallites of 10 healthy controls increased by approximately $800 \mathrm{~nm}$ from about $216 \mathrm{~nm}$ to $1010 \mathrm{~nm}$.

(2) The size distribution of urinary crystallites of one representative calcium oxalate monohydrate (COM) patient and one healthy control was shown in Figure 2 . The $\bar{d}$ values of the urinary crystallites of $\mathrm{CaO} x$ calculi patients grew from $267 \mathrm{~nm}$ to $3251 \mathrm{~nm}$ when the pore size of the membranes increased from $0.22 \mu \mathrm{m}$ to $10 \mu \mathrm{m}$ (Figure 2(a)). However, the $\bar{d}$ values of the urinary crystallites of the controls just increased from $184 \mathrm{~nm}$ to $1068 \mathrm{~nm}$ (Figure 2(b)).

(3) A small size difference between the two types of urinary crystallites was observed after filtration of the urine through a filter membrane of $0.22 \mu \mathrm{m}$ and $0.45 \mu \mathrm{m}$. However, the average size of the urinary crystallites of the calculi patients was significantly larger than that of the controls after urine filtration through membranes of $1.2,3.0$, and $10.0 \mu \mathrm{m}$. The difference between the sizes of the two types of urinary crystallites reached $1943 \mathrm{~nm}$ when the pore size was $10 \mu \mathrm{m}$. This result indicated that the quantity of largersized urinary crystallites of the $\mathrm{CaO} x$ calculi patients was significantly higher than that of the controls.

(4) The particle size of urinary crystallites was much smaller than the corresponding membrane pore size. For instance, the $\bar{d}$ value of urinary crystallites of the patients was $3251 \mathrm{~nm}$, whereas the controls was $1068 \mathrm{~nm}$ after filtration through a filter membrane 


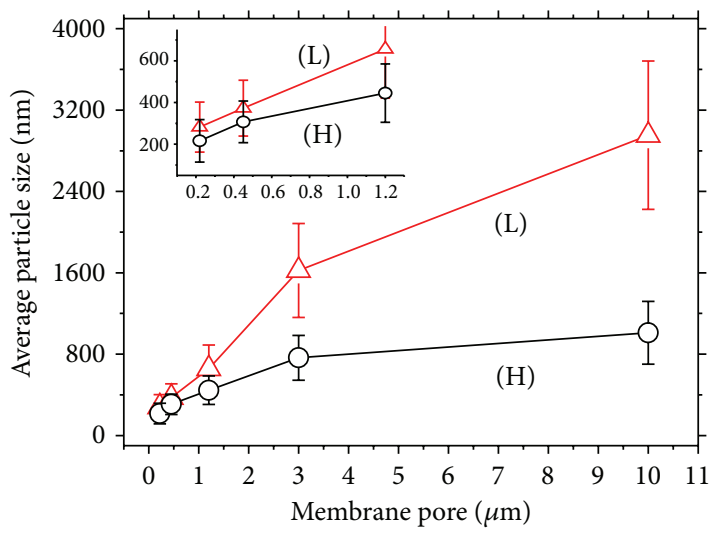

(a)

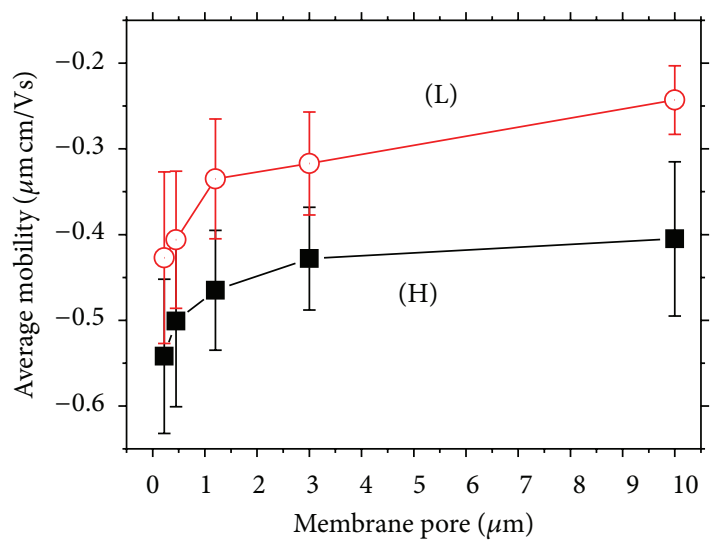

(c)

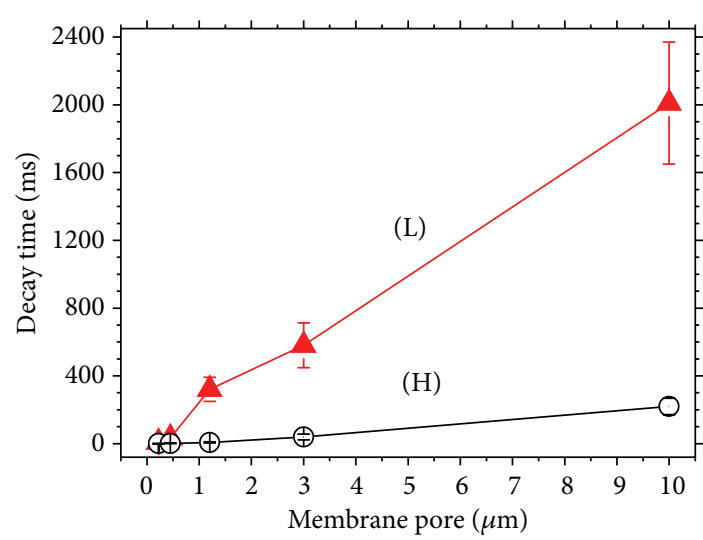

(e)

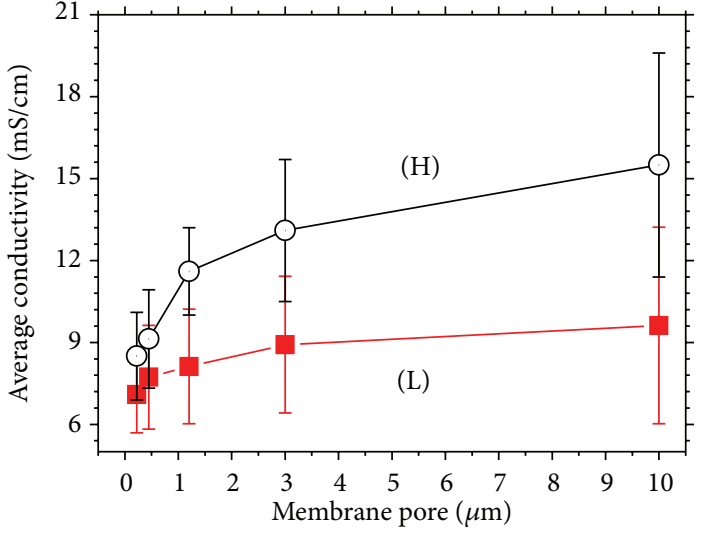

(b)

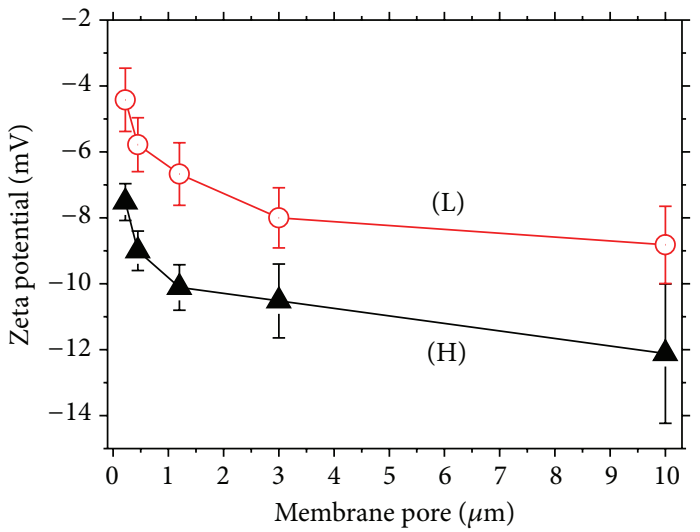

(d)

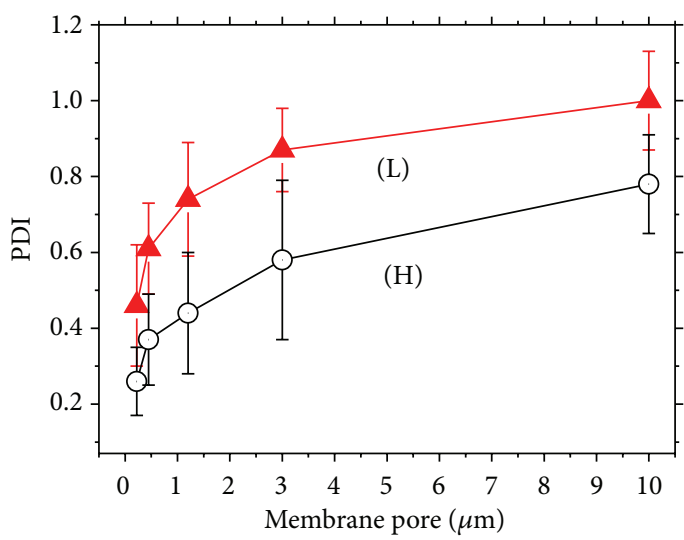

(f)

FIGURE 1: Property comparison of urinary crystallites from ten calculi patients and ten healthy controls filtered through membranes with different pore sizes. (L) means calculi patients and (H) means healthy controls. (a) Average size; (b) conductivity; (c) mobility; (d) zeta potential; (e) decay time ( $\left.T_{a}\right)$; (f) polydispersity index (PDI). The error bars showed the range of values obtained among the 10 patients and 10 healthy controls.

with a pore size of $10 \mu \mathrm{m}$. Therefore, the crystallite greater than $4 \mu \mathrm{m}$ was hard to pass through the filter membrane of $10 \mu \mathrm{m}$. This result will help select the appropriate pore size of the membrane in the future study of crystallite properties.

(5) Considering that the growth of urinary crystallites in urine is a very slow process, the agglomeration of urinary crystallites can be rapidly completed in a short time [12, 16]. Figure 1(a) shows that urinary crystallites of the calculi patients were easier to aggregate than those of the healthy controls. Furthermore, the small-sized urinary crystallites of the patients were easier to aggregate than those of the controls. Therefore, the rapid aggregation of urinary crystallites may be the key factor affecting urolith formation. 


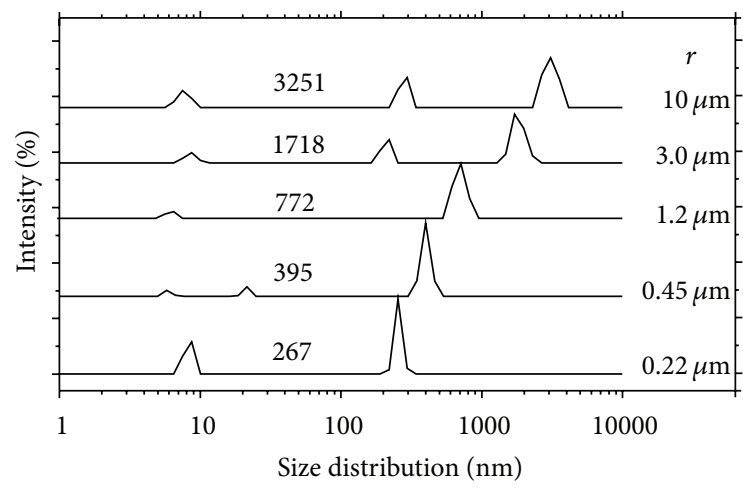

(a)

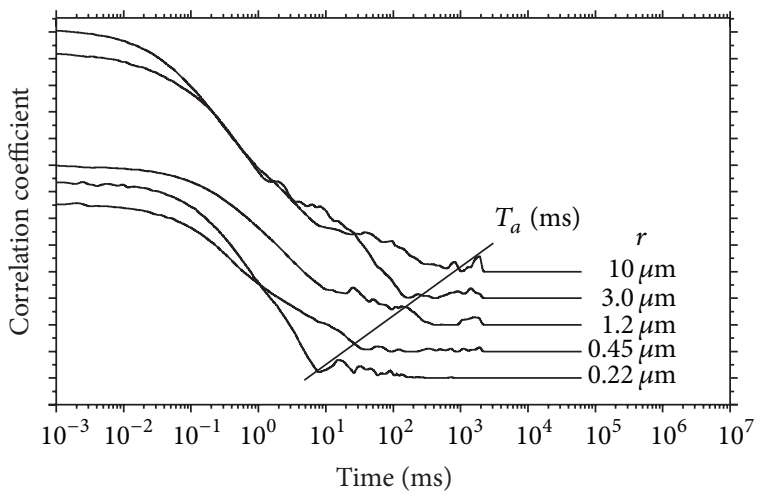

(c)

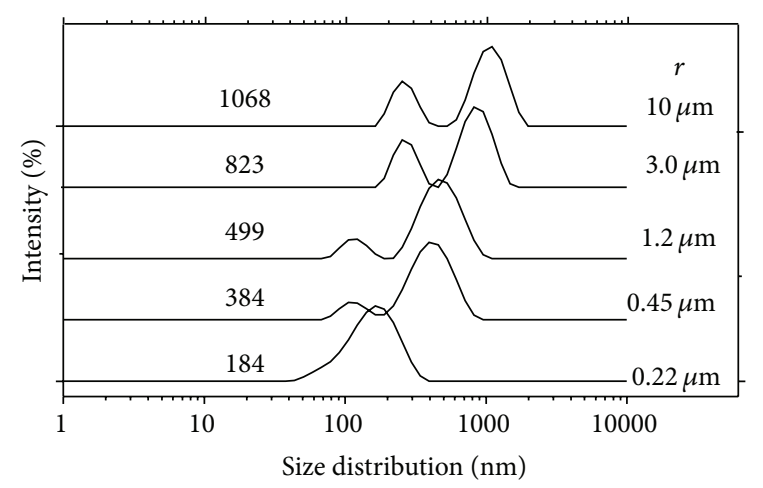

(b)

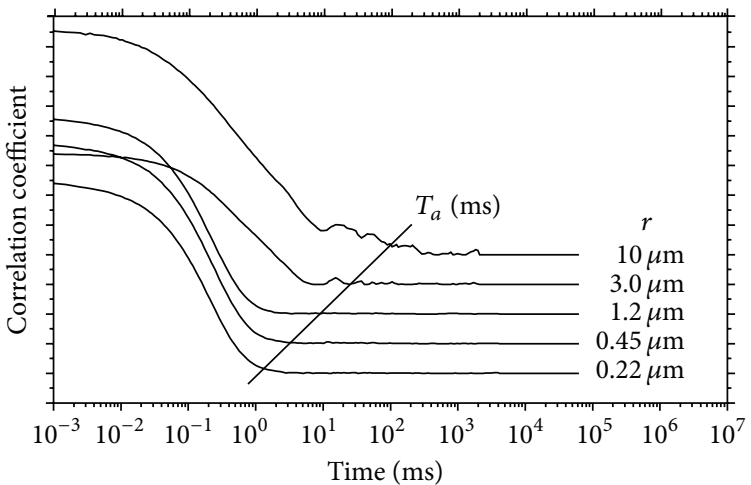

(d)

FiguRE 2: Size distribution ( $a, b)$ and autocorrelation curves $(c, d)$ of urinary crystallites of one calculi patient and one healthy control filtered through membranes with different pore sizes. (a, c) Patient with COM stones. (b, d) Healthy control. $r$ means microporous membrane pore size.

\subsection{Conductivity and Mobility Changes in the Different Sizes of Urinary Crystallites}

3.2.1. Conductivity Changes. Urine conductivity depends on the charge and concentration of the charged particles of different sizes in urine $[17,18]$. Figure 1(b) compares the conductivity changes in different particle sizes between the two types of urinary crystallites. The conductivity of urinary crystallites from calculi patients was lower than that of the controls.

The urine conductivity changes reflect the variations of renal concentration function. Lithiasis may increase the intrapelvic pressure and renal intracapsular pressure, which will decrease the effective filtration pressure and glomerular filtration rate, leading to swelling of the kidney tubules around the histogenesis, which would prevent urine concentration and eventually reduce the urine ion concentration [19]. Therefore, the urine conductivity of patients with stones was significantly lower than that of the controls.

The conductivity of the two kinds of urinary crystallites increased with increased pore size of the microporous membranes. However, the conductivity of the healthy controls was increased significantly. This result may be related to the increased number of crystallites after the increased pore size of the microporous membranes. In addition, the large-sized urinary crystallites were able to adsorb more charges [20], thereby increasing the concentration of the anion material in the urine of the controls.

3.2.2. Mobility. The mobility $(\mu)$ of the controls was more negative than that of the calculi patients (Figure 1(c)). The absolute value of mobility in the two kinds of urinary crystallites decreased with increased pore size of the microporous membrane. The mobility is greater when the particle velocity is faster [18]. Considering that the size of the urinary crystallites of the controls was smaller than that of the calculi patients (Figure 1(a)), the particle velocity of the controls was faster; therefore the absolute value of its mobility was greater.

\subsection{Influence of Pore Size of the Filtration Membrane on $\zeta$}

3.3.1. $\zeta$ of the Healthy Controls Is More Negative Than That of the Patients. $\zeta$ can be approximately expressed by the surface potential of uniformly charged spherical particles as follows: $\zeta=4 \pi(\sigma / \varepsilon \kappa)$, where $\sigma$ is the surface charge density of the particles, $\varepsilon$ is urine dielectric constant, and $\kappa$ is Debye-Hückel constant [20]. The $\zeta$ of the urinary crystallites is proportional to $\sigma$. The $\sigma$ is related to the concentration of the anionic inhibitors in the urine and the adsorption anionic ability of the urinary crystallites.

The healthy controls had more concentrated or more active inhibitory substances than the $\mathrm{CaO} x$ calculi patients; 


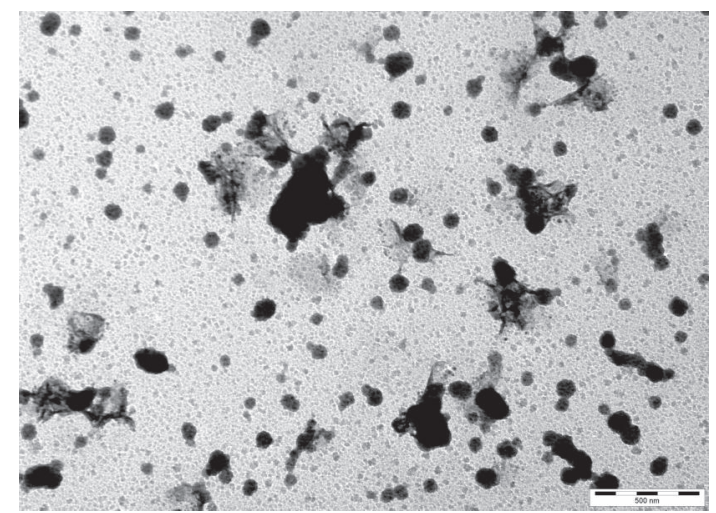

(a)

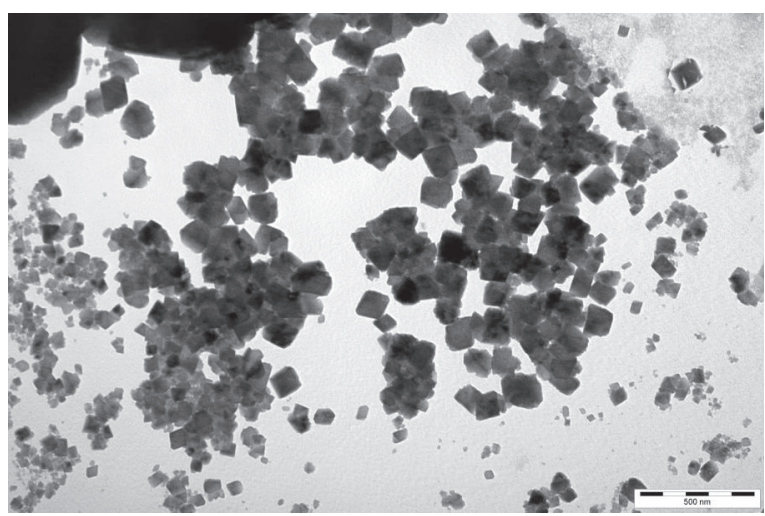

(b)

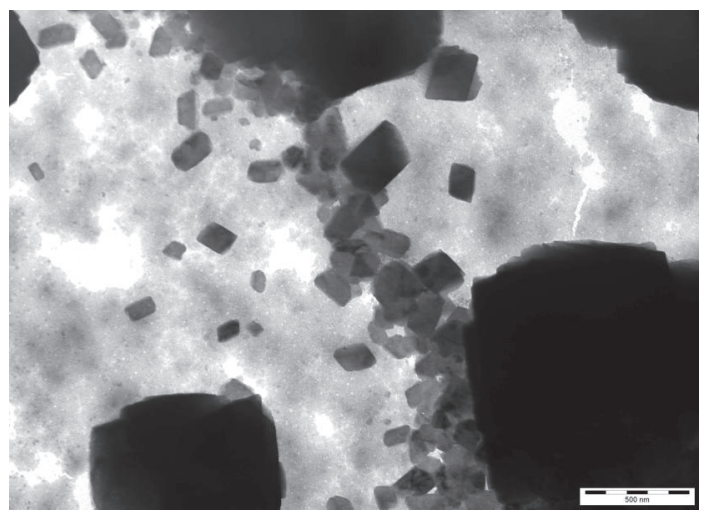

(c)

FIGURE 3: TEM images of urinary crystallites of calculi patient after filtration through membranes with different pore sizes: (a) $0.45 \mu \mathrm{m}$; (b) $1.2 \mu \mathrm{m}$; (c) $3.0 \mu \mathrm{m}$. The bars: $500 \mathrm{~nm}$. This figure showed that the particle size of the urinary crystallites of CaOx calculi patients increased gradually and distributed very unevenly with increased pore size. Large-sized crystallites in the patients were formed.

thus $\zeta$ of the healthy controls was more negative than that of the patients (Figure $1(\mathrm{~d})$ ). For example, the urinary gycosaminoglycans (GAGs) concentration of 10 patients and 10 controls in this study was $5.18 \pm 0.82$ and $9.80 \pm 1.83 \mathrm{mg} / \mathrm{L}$, respectively, and the content of citrate was $264 \pm 74$ and 348 $\pm 82 \mathrm{mg} / \mathrm{L}$, respectively [13].

In addition to the inhibitors and crystallite size in urine, the difference in the surface charge density may be due to different surface chemistry. For example, the calcium oxalate dihydrate (COD) crystals are more frequently observed in healthy urine and COD has less charge on surface, while the aggregated COM crystals are more frequently observed in the urine of lithogenic patients and COM has much positive charges on surface [6].

\subsection{2. $\zeta$ Becomes Negative with Increased Membrane Pore} Size. Figure $1(\mathrm{~d})$ also shows that the average $\zeta$ of the urinary crystallites of 10 cases of controls decreased from $-7.52 \mathrm{mV}$ to $-12.12 \mathrm{mV}$ with increased filtration membrane aperture from $0.22 \mu \mathrm{m}$ to $10 \mu \mathrm{m}$, respectively. However, $\zeta$ of the urinary crystallites of 10 patients decreased from $-4.42 \mathrm{mV}$ to $-8.82 \mathrm{mV}$, respectively. The adsorption capacity of the microsized urinary crystals to anions was greater than that of the nano-sized urinary crystallites. The smallsized nanocrystallites may have many grain boundaries and numerous lattice defects [21]; thus they are weak in adsorbing anions and have few surface charges [20,22], resulting in the smaller absolute value of $\xi$.

The value of $\zeta$ directly reflects the electrostatic repulsion between the urinary crystallites. If $\zeta$ is more negative, that is, its absolute value is greater, the electrostatic repulsion between the urinary crystallites is greater and the aggregation of urinary crystallites is harder, which is more beneficial to inhibit stone formation.

3.4. Light Intensity Autocorrelation Curve, PDI, and Decay Time. The light intensity autocorrelation function of the urinary crystallites can reflect the size of crystallites and the stability of urine. The urine is more stable when the autocorrelation curve is smoother $[23,24]$. The decay time would be shorter when the autocorrelation curve decay is faster, which would indicate that the urinary crystallite size was even and the urinary system was stable.

(1) Figures 2(c) and 2(d) show the light intensity autocorrelation curve of the two types of urinary crystallites in Figures 2(a) and 2(b), respectively. The autocorrelation curve of the urinary crystallites of the patients became rough, the curve attenuation became slower, and the volatility increased when the pore size of the 


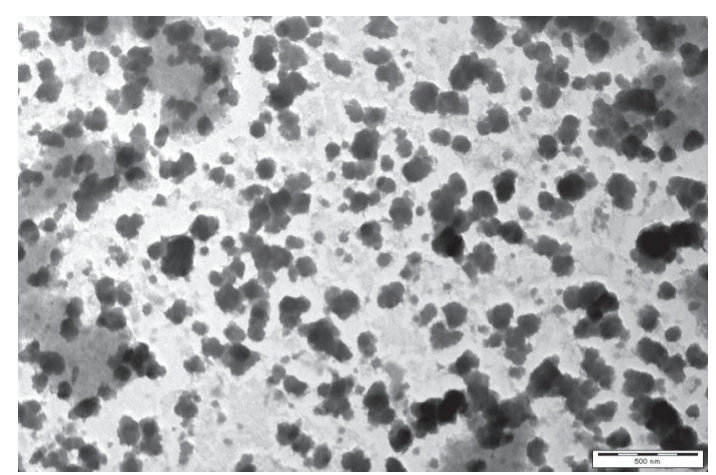

(a)

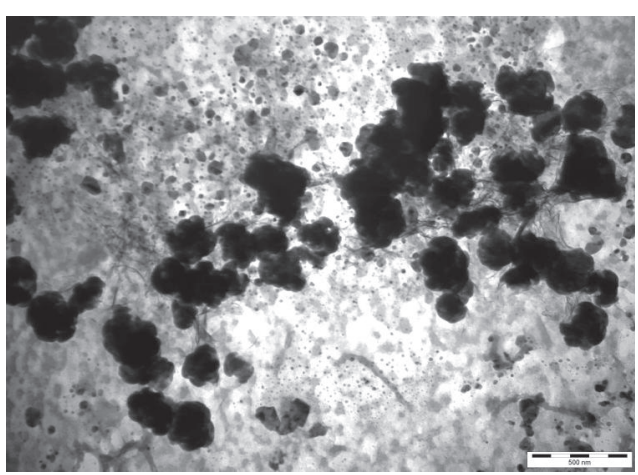

(b)

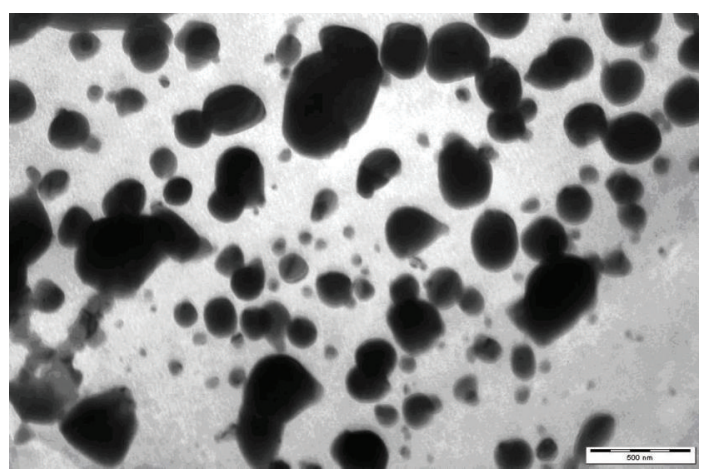

(c)

FIGURE 4: TEM images of urinary crystallites of healthy control after filtration through membranes with different pore sizes: (a) $0.45 \mu \mathrm{m}$; (b) $1.2 \mu \mathrm{m}$; (c) $3.0 \mu \mathrm{m}$. The bars: $500 \mathrm{~nm}$. This figure showed that the small-sized urinary crystallites of the controls were significantly higher than those of the patients. The particle size distribution of urinary crystallites in the controls is much narrower than that of the patients.

microporous membrane was increased from $0.22 \mu \mathrm{m}$ to $10 \mu \mathrm{m}$ (Figure 2(c)). Accordingly, the decay time $\left(T_{a}\right)$ increased significantly by about $2000 \mathrm{~ms}$ from $8.1 \mathrm{~ms}$ to $2010 \mathrm{~ms}$ (Figure 1(e)), which showed that the urine was unstable. The autocorrelation curve of the urinary crystallites of the controls was very smooth (Figure 2(d)), and the decay time only increased by about $200 \mathrm{~ms}$ from $0.32 \mathrm{~ms}$ to $220 \mathrm{~ms}$. The urinary crystallites of the patients would be continuously magnified with increased membrane size because of the larger proportion of large-sized crystallites. The light scattering intensity is stronger when the particle size is greater. Therefore, the light scattering intensity and decay time of the crystallites of the patients were greater than those of the controls.

(2) The polydispersity index (PDI) is a width parameter that characterizes the distribution range of particle size. The distribution range of particle size is narrower when the PDI value is smaller. Thus, the particle would be more uniform. The PDI of the urinary crystallites of the patients increased from 0.46 to 0.91 when the pore size of the microporous membrane was increased from $0.22 \mu \mathrm{m}$ to $10 \mu \mathrm{m}$ (Figure 1(f)), which shows the instability of these urine samples. However, the PDI of the urinary crystallites of the controls increased from 0.26 to 0.78 . The decreased PDI of the controls indicated that the distribution range of their urinary crystallites was narrower and the particle size tended to be uniform $[12,25,26]$. These results were consistent with the results of Figure 2.

These results show that the urinary crystallite size of $\mathrm{CaO} x$ calculi patients was uneven, the urinary system was unstable, and the urinary crystallites were prone to aggregate and coagulate, which would lead to an increased risk of urinary stone formation.

3.5. Morphology of Different Sizes of Urinary Crystallites. The morphology of urinary crystallites in the $\mathrm{CaO} x$ calculi patients and controls after filtration through filter membranes with different pore sizes was studied by TEM. The representative TEM images are shown in Figures 3 and 4 . The particle size of the urinary crystallites of the calculi patients gradually increased and distributed very unevenly with increased pore size. For example, the urinary crystallite diameter ranged from $30 \mathrm{~nm}$ to $300 \mathrm{~nm}$ after filtration through a $0.45 \mu \mathrm{m}$ membrane (Figure 3(a)) and between $100 \mathrm{~nm}$ and $2700 \mathrm{~nm}$ after filtration through a $3 \mu \mathrm{m}$ membrane (Figure $3(\mathrm{c})$ ). By contrast, the urinary crystallite diameter from a healthy volunteer ranged from $100 \mathrm{~nm}$ to $200 \mathrm{~nm}$ after filtration through a $0.45 \mu \mathrm{m}$ membrane filter (Figure $4(\mathrm{a})$ ) and between $100 \mathrm{~nm}$ and $700 \mathrm{~nm}$ after filtration through a $3 \mu \mathrm{m}$ membrane filter (Figure 4(c)). The number of large-sized crystallites of 
TABle 1: Properties comparison of urinary crystallites filtered through membranes with different pore sizes from ten CaOx calculi patients and ten healthy controls.

\begin{tabular}{|c|c|c|c|c|c|}
\hline Membranes pore sizes $/ \mu \mathrm{m}$ & 0.22 & 0.45 & 1.2 & 3 & 10 \\
\hline \multicolumn{6}{|l|}{ Calculi patients $(n=10)$} \\
\hline Average size by NSA ${ }^{* 1} / \mathrm{nm}$ & $282 \pm 120$ & $373 \pm 134$ & $656 \pm 235$ & $1624 \pm 462$ & $2953 \pm 730$ \\
\hline Average size by TEM/nm & & $120 \pm 108$ & $250 \pm 210$ & $460 \pm 376$ & \\
\hline Zeta potential $^{* 2} / \mathrm{mV}$ & $-4.42 \pm 0.96$ & $-5.78 \pm 0.82$ & $-6.67 \pm 0.95$ & $-8 \pm 0.91$ & $-8.82 \pm 1.17$ \\
\hline \multicolumn{6}{|l|}{ Healthy controls $(n=10)$} \\
\hline Average size by NSA ${ }^{* 1} / \mathrm{nm}$ & $216 \pm 102$ & $307 \pm 100$ & $445 \pm 140$ & $764 \pm 221$ & $1010 \pm 309$ \\
\hline Average size by TEM/nm & & $120 \pm 49$ & $180 \pm 79$ & $250 \pm 121$ & \\
\hline Zeta potential $^{* 2} / \mathrm{mV}$ & $-7.52 \pm 0.56$ & $-9.00 \pm 0.60$ & $-10.11 \pm 0.69$ & $-10.52 \pm 1.12$ & $-12.12 \pm 2.11$ \\
\hline
\end{tabular}

${ }^{* 1}$ These data were obtained from Figure 1(a), which detected using nanoparticle size analyzer.

${ }^{* 2}$ The data were obtained from Figure 1(d).

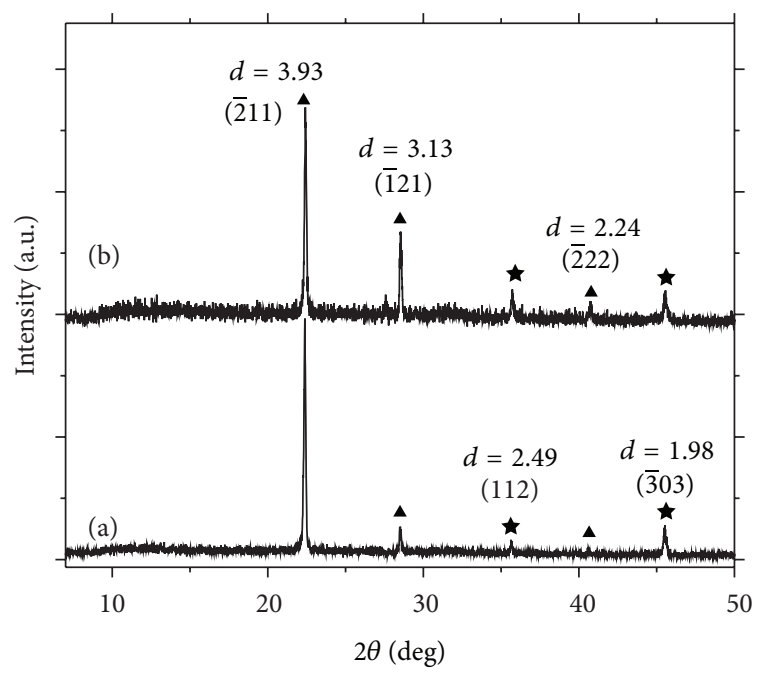

FIGURE 5: XRD patterns of urinary crystallites of calculi patients after filtration through membranes with different pore sizes: (a) $1.2 \mu \mathrm{m}$; (b) $3.0 \mu \mathrm{m}$. Black star: COM; $\mathbf{\Delta}$ : uric acid. This figure demonstrated that the components of nanosized urinary crystallites were similar to those of the microsized crystallites.

the calculi patients was higher than that of the controls. The TEM results were consistent with the nanoparticle size analysis results (Figure 1(a)).

The crystallites from patients with $\mathrm{CaO} x$ stones exhibited sharp edges and corners (Figure 3). However, few crystallites with sharp edges and corners were observed for healthy control subjects (Figure 4). Changes in the appearance and size of urinary crystallites were due to the following reasons. (1) The urine of the control subjects contains a high concentration of inhibitors such as citrate and GAGs and so forth, which are chelating agents. For example, $1 \mu \mathrm{mol}$ of chondroitin sulfate (one of the eight GAGs) disaccharide unit can combine $0.757 \mu \mathrm{mol}$ of free $\mathrm{Ca}^{2+}$ ions [27]. When these inhibitors combine with $\mathrm{Ca}^{2+}$ ions in urine to form soluble calcium salts, $\mathrm{Ca}^{2+}$ ion concentration and $\mathrm{CaO} x$ supersaturation in urine were reduced. Thus, the growth and aggregation of $\mathrm{CaO} x$ crystallites were inhibited, and the size of urinary crystallites was reduced. (2) Urine inhibitors can maintain complexation-dissociation equilibria with $\mathrm{Ca}^{2+}$ ions on surface of $\mathrm{CaO} x$ crystallites, particularly the $\mathrm{Ca}^{2+}$ ions on the peripheries and edges of crystallites. That is, the $\mathrm{Ca}^{2+}$ ions on crystallite surfaces were ceaselessly dissolved by citrate and GAGs; at the same time, the dissolved $\mathrm{Ca}^{2+}$ ions also continuously precipitated on the surface of crystallites. This continuous precipitation-dissolution process produced blunt edges and corners of crystallites (Figure 4).

Image Pro Plus 5.02 (Media Cybernetics, USA) was used to analyze the diameter and count the number of particles in the transmission electron microscopy (TEM) images. Table 1 lists the particle sizes of urinary crystallites measured by TEM and nanoparticle size analysis (NSA). The TEM results were less than the NSA results. The difference was due to the different experimental conditions. TEM data were obtained from the samples after drying in a vacuum, whereas NSA data were obtained in solution. The particle diameter detected by NSA was the hydrodynamic diameter of urinary crystallites $[28,29]$. Considering that the light scattering intensity of large-sized crystallites is exponentially increased compared with that of small-sized crystallites, we found that the presence of a small amount of large crystallites likely caused a significant increase of the detection values when NSA was used [26]. Therefore, the deviation of particle size detected by NSA is possibly larger than that of TEM. In general, the TEM results were similar to the actual results. This difference between the TEM and NSA measurement results is consistent with those reported in a previous study [30].

The particle size distribution of urinary crystallites in the patients $(5 \mathrm{~nm}$ to $3600 \mathrm{~nm})$ is much wider than that of the controls ( $30 \mathrm{~nm}$ to $1300 \mathrm{~nm}$ ). According to Ostwald ripening theory, the wide range of particle size distribution of urinary crystallites is conducive to dissolve the small-sized urinary crystallites and further increase the large-sized urinary crystallites, which enables the system to maintain a lower energy state. As size of $\mathrm{CaO} x$ particles decreases from $1 \mu \mathrm{m}$ to 0.1 and $0.01 \mu \mathrm{m}$, its solubility will increase from $0.6 \%$ to $6.3 \%$ and $84 \%$, respectively [31]. The particle size distribution in the controls is narrow (Figure 1(a), Table 1) and the urine is stable, which is beneficial to inhibit urinary stone formation.

3.6. Detection of Urinary Crystallite Components. X-ray diffraction (XRD) analysis revealed no significant differences in the urinary crystallite components after filtration through 


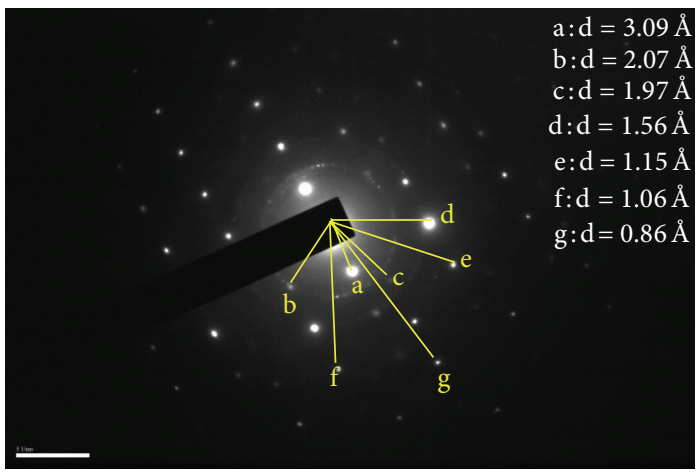

(a)

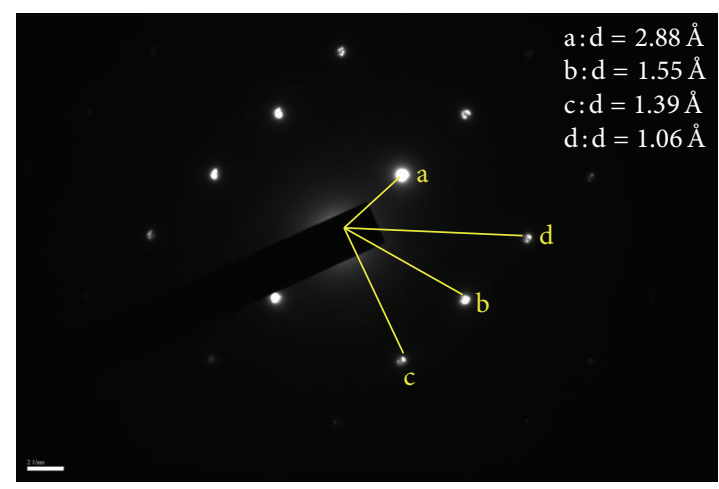

(b)

FIgURE 6: SAED images of urinary nanocrystallites in patients with $\mathrm{CaO} x$ calculi.

membranes with different pore sizes. Figure 5 shows one representative case. The corresponding diffraction peaks located at $3.93,3.13$, and $2.24 \AA$ were assigned to the (211), (121), and (222) of uric acid, respectively. The diffraction peaks located at 2.49 and $1.98 \AA$ were assigned to the (112) and $(\overline{3} 03)$ of COM, respectively. The main components of the crystallites were uric acid and COM. That is, the XRD results demonstrated that the nanosized urinary crystallite components were similar to the microsized crystallite components.

3.7. SAED Analysis of Urinary Crystallites. The components of urinary nanocrystallites in the patient's urines were characterized using selected area electron diffraction (SAED). Figure 6 showed the two representative SAED images. A series of diffraction points or diffraction rings appeared, which demonstrated that these substances were single crystal or polycrystalline $[32,33]$. The diffraction data were indexed and compared with the ASTM standard curve; the diffraction points or diffraction rings were attributed. In Figure 6(a) we detected the peak at $d=3.09 \AA$, which was assigned to ( $\overline{1} 21$ ) plane of uric acid; the peaks at $d=2.07,1.97,1.56,1.15,1.06 \AA$, which were assigned to (321), ( $\overline{3} 03),(334),(1040)$, and (1060) planes of COM, respectively. In Figure 6(b) we detected the peaks at $d=2.88,1.55 \AA$, which were assigned to (0210), (517) planes of $\mathrm{CaP}$; the peaks at $d=1.39,1.06 \AA$, which were assigned to (604), (1060) planes of COM, respectively. The formation of $\mathrm{CaO} x$ calculi was closely related to the presence of UA and calcium phosphate crystallites in urine. This result provided new evidence of uric acid or calcium phosphate as nidus to induce calcium oxalate stone formation.

\section{Conclusions}

The properties of different size ranges of urinary crystallites between $\mathrm{CaO} x$ calculi patients and healthy controls were compared. The urinary crystallites of the calculi patients were much easy to aggregate than those of controls after filtration through filter membranes from $0.22 \mu \mathrm{m}$ to $0.45,1.2,3$, and $10 \mu \mathrm{m}$. The number of large-sized crystallites of the controls was significantly less than that of the calculi patients. The main components of the nanosized urinary crystallites in patients with $\mathrm{CaO} x$ calculi were $\mathrm{CaO} x$ monohydrate $(\mathrm{COM})$, uric acid, and $\beta$-calcium phosphate, and these components were basically similar to those of the microsized urinary crystallites. The urinary crystallites of the calculi patients were easier to aggregate than those of the healthy controls, and the small-sized urinary crystallites were much easier to agglomerate. Therefore, the rapid aggregation of urinary crystallites may be the key factor affecting urolith formation. The urinary crystallite size of $\mathrm{CaO} x$ calculi patients was uneven, the urinary system was unstable, and the urinary crystallites were prone to aggregate and coagulate, which would lead to an increased risk of urinary stone formation.

\section{Conflict of Interests}

The authors declare that there is no conflict of interests regarding the publication of this paper.

\section{Acknowledgment}

This research work was supported by the Natural Science Foundation of China $(81170649,21371077)$.

\section{References}

[1] M. Daudona and P. Jungers, "Clinical value of crystalluria and quantitative morphoconstitutional analysis of urinary calculi," Nephron: Physiology, vol. 98, no. 2, pp. p31-p36, 2004.

[2] S. Verdesca, G. B. Fogazzi, G. Garigali, P. Messa, and M. Daudon, "Crystalluria: prevalence, different types of crystals and the role of infrared spectroscopy," Clinical Chemistry and Laboratory Medicine, vol. 49, no. 3, pp. 515-520, 2011.

[3] J.-M. Ouyang, Z.-Y. Xia, G.-N. Zhang, and H.-Q. Chen, “The nanocrystallites in urine and its relationship with formation of kidney stones," Reviews in Inorganic Chemistry, vol. 32, no. 2-4, pp. 101-110, 2012.

[4] R. S. Bareeva, I. I. Turkovsky, L. M. Molodkina, B. A. Paramonov, K. P. Pomorsky, and A. B. Samatigo, "UHFdielectrometry of the urine in prognosis of aggregation stability," Bulletin of Experimental Biology and Medicine, vol. 145, no. 4, pp. 433-435, 2008. 
[5] H. Peng, J.-M. Ouyang, X.-Q. Yao, and R.-E. Yang, "Interaction between sub-micron COD crystals and African green monkey renal epithelial cell," International Journal of Nanomedicine, vol. 7, no. 8, pp. 4727-4737, 2012.

[6] J.-Y. He, S.-P. Deng, and J.-M. Ouyang, "Morphology, particle size distribution, aggregation, and crystal phase of nanocrystallites in the urine of healthy persons and lithogenic patients," IEEE Transactions on Nanobioscience, vol. 9, no. 2, pp. 156-163, 2010.

[7] M. Daudon, C. Hennequin, G. Boujelben, B. Lacour, and P. Jungers, "Serial crystalluria determination and the risk of recurrence in calcium stone formers," Kidney International, vol. 67, no. 5, pp. 1934-1943, 2005.

[8] Y. M. Fazil Marickar and A. Salim, "Clinical risk index in urolithiasis," Urological Research, vol. 37, no. 5, pp. 283-287, 2009.

[9] M. Robert, A.-M. Boularan, O. Delbos, L. Monnier, and D. Grasset, "Evaluation of the risk of stone formation: study on crystalluria in patients with recurrent calcium oxalate urolithiasis," European Urology, vol. 29, no. 4, pp. 456-461, 1996.

[10] B.-S. Gui, Z.-J. Huang, X.-J. Xu, M.-R. Li, J.-Y. He, and J.-M. Ouyang, "Measurement of urine crystallites and its influencing factors by nanoparticle size analyzer," Journal of Nanoscience and Nanotechnology, vol. 10, no. 8, pp. 5232-5241, 2010.

[11] G.-N. Zhang, J.-M. Ouyang, J.-F. Xue, and Y.-F. Shang, "Property changes of urinary nanocrystallites and urine of uric acid stone formers after taking potassium citrate," Materials Science and Engineering C: Materials for Biological Applications, vol. 33, no. 7, pp. 4039-4045, 2013.

[12] J.-Y. He, J.-M. Ouyang, and R.-E. Yang, "Agglomeration of urinary nanocrystallites: key factor to formation of urinary stones," Materials Science and Engineering C: Materials for Biological Applications, vol. 30, no. 6, pp. 878-885, 2010.

[13] C.-Y. Duan, Z.-Y. Xia, G.-N. Zhang, B.-S. Gui, J.-F. Xue, and J.-M. Ouyang, "Changes in urinary nanocrystallites in calcium oxalate stone formers before and after potassium citrate intake," International Journal of Nanomedicine, vol. 8, pp. 909-918, 2013.

[14] Z.-Y. Xia, Y.-M. Ding, and J.-M. Ouyang, "Comparison of urinary crystallites from patients with renal calculi with that from healthy subjects," Advanced Materials Research, vol. 554556, pp. 1738-1741, 2012.

[15] Z.-J. Huang, J.-J. Li, J.-Y. He, and J.-M. Ouyang, "Study on nanoand microcrystallites in the urines of calcium oxalate stone formers," Spectroscopy and Spectral Analysis, vol. 30, no. 7, pp. 1913-1917, 2010.

[16] J. A. Wesson, V. Ganne, A. M. Beshensky, and J. G. Kleinman, "Regulation by macromolecules of calcium oxalate crystal aggregation in stone formers," Urological Research, vol. 33, no. 3, pp. 206-212, 2005.

[17] A. V. Popov and E. Kitaeva, "Changes of bile and urine electrical conductivity in patients with biliary tracts' pathology," in Proceedings of the 13th International Conference on Electrical Bioimpedance and the 8th Conference on Electrical Impedance Tomography (ICEBI '07), pp. 644-646, Springer, Berlin, Germany, September 2007.

[18] Y. M. Fazil Marickar, "Electrical conductivity and total dissolved solids in urine," Urological Research, vol. 38, no. 4, pp. 233-235, 2010.

[19] F. Manoni, L. Fornasiero, M. Ercolin et al., "Laboratory diagnosis of renal failure: urine conductivity and tubular function," Italian Journal of Urology and Nephrology, vol. 61, no. 1, pp. 1720, 2009.
[20] N. Pawar and H. B. Bohidar, "Surface selective binding of nanoclay particles to polyampholyte protein chains," Journal of Chemical Physics, vol. 131, no. 4, Article ID 045103, 2009.

[21] Q. Chen and S. R. Yun, "Synthesizing nano-sized diamond through explosive detonation and its application." Journal of Synthetic Crystals, vol. 29, no. 1, pp. 90-93, 2000 (Chinese).

[22] G. Sonavane, K. Tomoda, and K. Makino, "Biodistribution of colloidal gold nanoparticles after intravenous administration: effect of particle size," Colloids and Surfaces B: Biointerfaces, vol. 66, no. 2, pp. 274-280, 2008.

[23] N. Laube, B. Mohr, and A. Hesse, "Laser-probe-based investigation of the evolution of particle size distributions of calcium oxalate particles formed in artificial urines," Journal of Crystal Growth, vol. 233, no. 1-2, pp. 367-374, 2001.

[24] J.-J. Li, S.-H. Hou, J.-Y. He, Z.-Y. Xia, and J.-M. Ouyang, "ל potential, autocorrelation curve and agglomeration of urinary crystallites with different size," Chinese Journal of Inorganic Chemistry, vol. 27, no. 12, pp. 2425-2430, 2011.

[25] D. L. Murphy, S. N. Beretvas, and K. A. Pituch, "The effects of autocorrelation on the curve-of-factors growth model," Structural Equation Modeling, vol. 18, no. 3, pp. 430-448, 2011.

[26] B. J. Frisken, "Revisiting the method of cumulants for the analysis of dynamic light-scattering data," Applied Optics, vol. 40, no. 24, pp. 4087-4091, 2001.

[27] J.-M. Ouyang, X.-Q. Yao, Z.-X. Su, and F.-Z. Cui, "Simulation of calcium oxalate stone in vitro," Science in China, Series B: Chemistry, vol. 46, no. 3, pp. 234-242, 2003.

[28] D. C. Prieve, J. D. Hoggard, R. Fu, P. J. Sides, and R. Bethea, "Two independent measurements of debye lengths in doped nonpolar liquids," Langmuir, vol. 24, no. 4, pp. 1120-1132, 2008.

[29] M. Wang, Q.-F. An, L.-G. Wu, J.-X. Mo, and C.-J. Gao, "Recent developments in the measurement of zeta-potential of membrane," Chinese Journal of Analytical Chemistry, vol. 35, no. 4, pp. 605-610, 2007.

[30] C. Volcke, S. Pirotton, C. Grandfils et al., "Influence of DNA condensation state on transfection efficiency in DNA/polymer complexes: an AFM and DLS comparative study," Journal of Biotechnology, vol. 125, no. 1, pp. 11-21, 2006.

[31] J.-M. Ouyang, M.-H. Wan, and N. Zhou, "Study of nanoparticles in urines by transmission electron microscopy," International Journal of Nanoscience, vol. 5, no. 6, Article ID 00513, pp. 769-773, 2006.

[32] E. M. Pouget, P. H. H. Bomans, J. A. C. M. Goos, P. M. Frederik, G. De With, and N. A. J. M. Sommerdijk, "The initial stages of template-controlled $\mathrm{CaCO}_{3}$ formation revealed by Cryo-TEM," Science, vol. 323, no. 5920, pp. 1455-1458, 2009.

[33] M. Marszaiek, "Application of optical microscopy and scanning electron microscopy to the study of stone weathering: a cracow case study," International Journal of Architectural Heritage, vol. 2, no. 1, p. 832, 2008. 

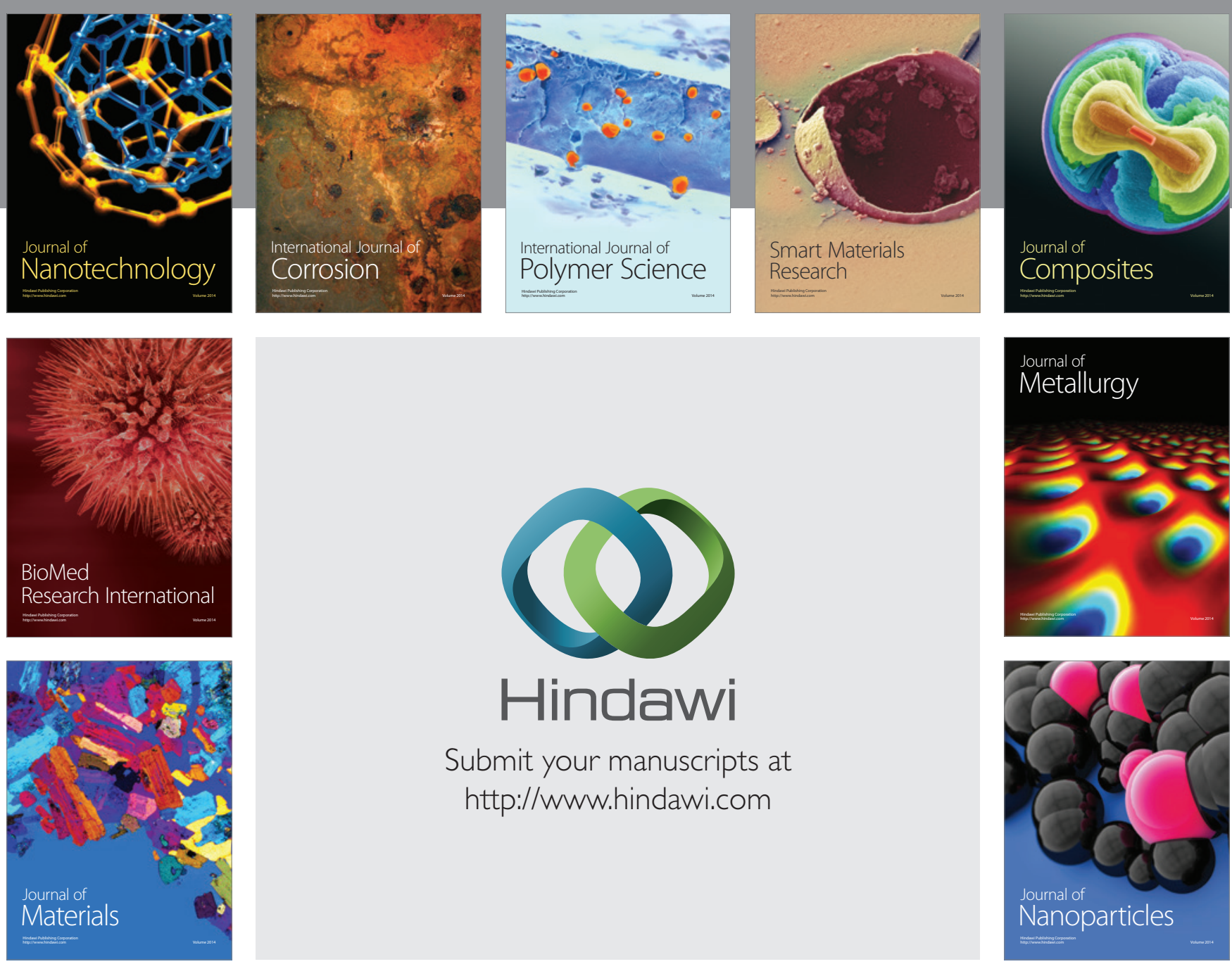

Submit your manuscripts at http://www.hindawi.com
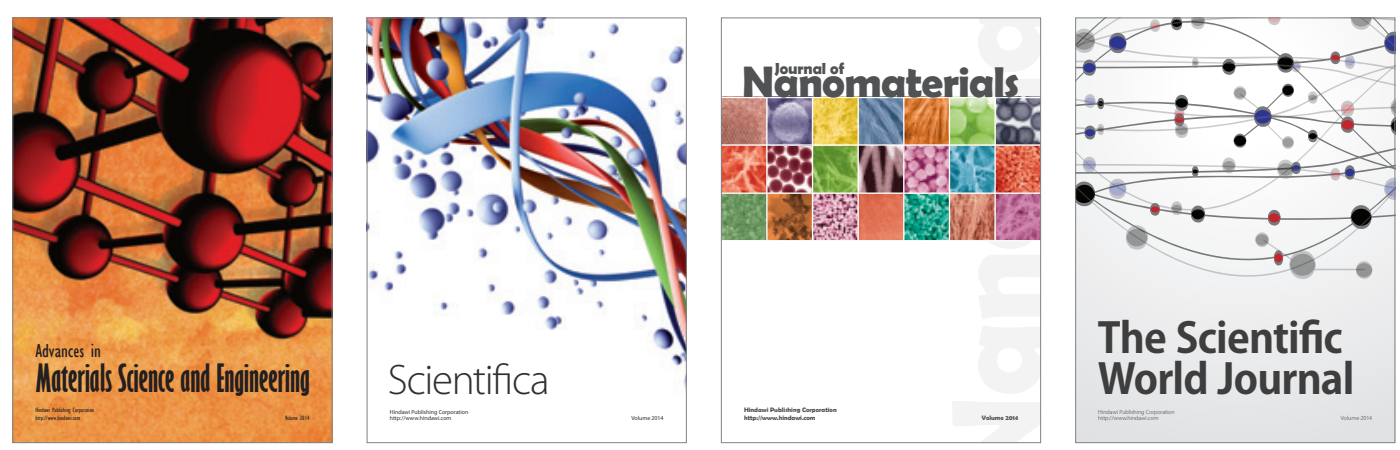

\section{The Scientific World Journal}
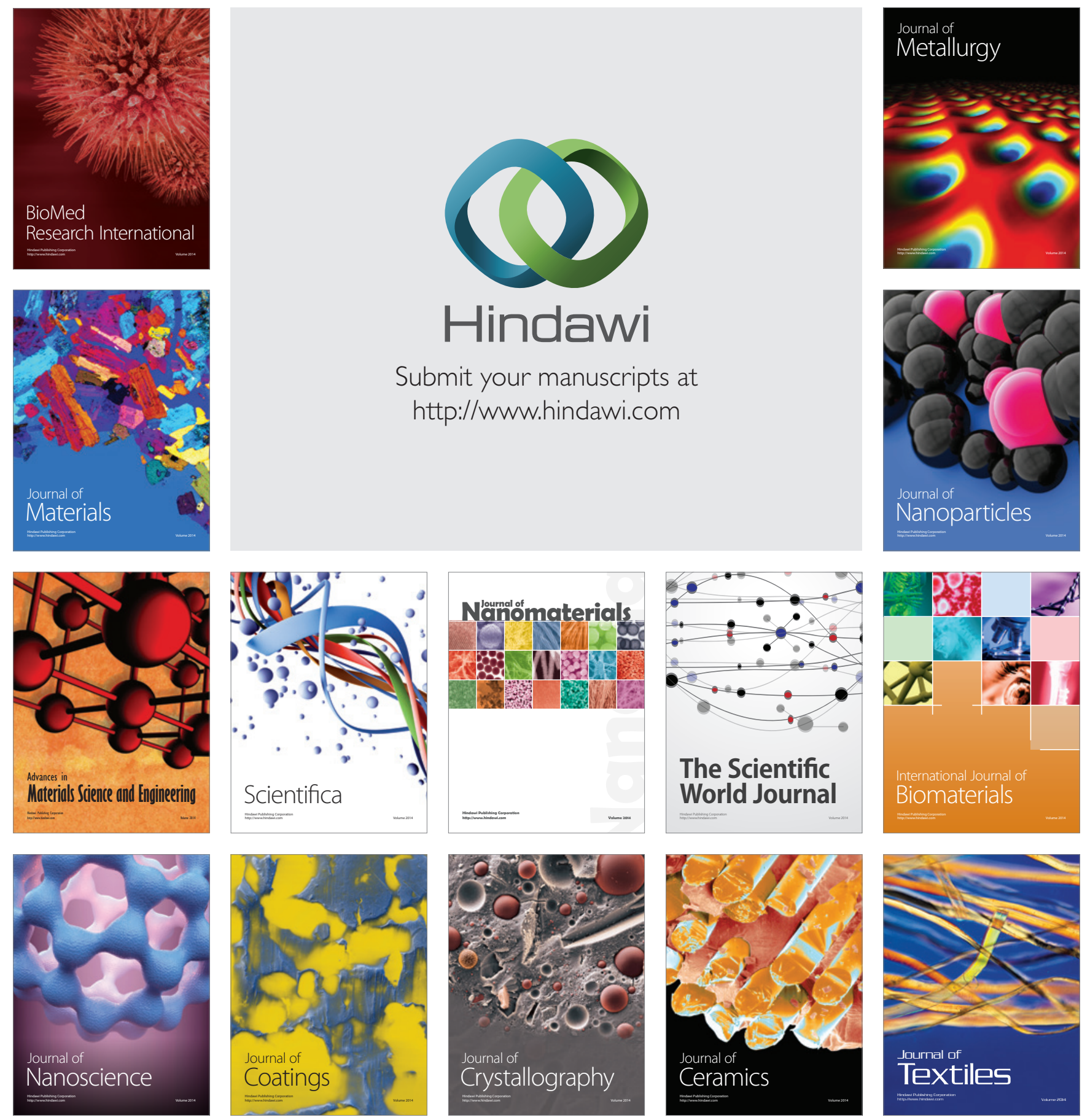